\title{
Aperture effects on charged particles in a magnetic field
}

\author{
E. Y. Wang ${ }^{\text {a) }}$, W. Q. Li, S. W. Lam and, N. Hershkowitz \\ Department of Nuclear Engineering and Engineering Physics, University of Wisconsin-Madison, \\ Wisconsin 53706
}

(Received 1 October 1990; accepted for publication 5 June 1991)

\begin{abstract}
In this paper we present investigations of two kinds of aperture effects on charged particles in the presence of a magnetic field. The first is an experimental study of the plasma potential profiles in front of an aperture. Plasma potential is measured with emissive probes. The interaction between the plasma and the solid wall of the aperture results in a nonuniform potential profile. Our experiments show that suitable aperture biasing can smooth the electric field in front of the aperture. The second is the transmission of electrons through a finite thickness aperture. The experimental results of the latter are compared to results of a single particle orbit model. It is shown that transmission measurements can be used to estimate the electron perpendicular energy in the magnetic field.
\end{abstract}

\section{INTRODUCTION}

Plasma flux analysis samples charged particles (ions or electrons) through a sampling aperture. After extraction from the plasma, the particle flux is either energy and/or mass-analyzed. Analysis of these data leads to estimates of plasma parameters. Flux analysis of plasma ions or electrons is not as straightforward as the analysis of neutrals, because of the presence of the electrostatic interaction between the sampling aperture and the plasma. Furthermore, when magnetic fields are present, the gyro-motion of charged particles in magnetic fields complicates the analysis. The charged particle flux which passes through an aperture depends on the plasma conditions in the vicinity of the aperture, the geometry and electrostatic potential of the aperture, the $B$ field, etc. In general particle distribution functions are distorted in passing through the aperture. In this paper, the effect of a finite thickness aperture on the transmission of charged particles is investigated. Although this problem is not solvable analytically in general, a few special cases of practical importance are well treated. Drawin ${ }^{1}$ has discussed conditions for extracting plasma ions from a glow discharge plasma. The results depend on the extraction aperture diameter $D$ and the plasma parameters, such as the relative magnitude of the ion mean-free-paths $\lambda_{i}$, the Debye shielding length $\lambda_{D}$, and the ion gyro-radius.

The simplest case is extraction of an unmagnetized plasma through a collisionless plasma sheath, which occurs when $\lambda_{i}>D, \lambda_{D}$. In this case, if the extraction aperture is biased negatively (or simply an aperture in the cathode if the plasma is a dc glow discharge), the sampled flux is described by the ion saturation current ${ }^{2} I$ $=0.6 e n\left(T_{e} / M_{i}\right)^{1 / 2}$, where $n, T_{e}$, and $M_{i}$ are the ion density, electron temperature, and mass of the positive ion species, respectively. The case of extraction through a collision dominated plasma sheath, (where $\lambda_{i}<\lambda_{D}, D$ ) is more complicated. Collision processes inside the plasma sheath may lead to a change of the distribution function of the sampled plasma particle flux.

Flux analysis of magnetized plasma is more difficult. Because of the confinement of charged particles by a magnetic field, the charged particles flux tends to diffuse along the magnetic field lines, but transverse diffusion is induced by space-charge effects, particle drift, or collisions. If the aperture axis is parallel to the magnetic field, the streaming flux will be sampled, whereas if the aperture axis is perpendicular to the magnetic field, the cross-field diffusive flux will be sampled. Here we shall concentrate our interest on the former. Accurate experimental analysis is somewhat difficult because of the following factors: (a) Sheath potential profiles existing in front of the aperture may alter the distribution function of the charged particles in the case of low temperature plasma; (b) the transmission of the aperture is energy-dependent because it has a finite thickness. Krawec ${ }^{3}$ had given an analysis of the problem for both thick and thin apertures and presented a set of numerical calculations in terms of the dimensionless parameters in the problem. (c) Space-charge effects may alter the distribution because of the presence of internal electric fields at higher plasma density.

In this paper we describe experimental investigations with emissive probes $^{2}$ of the plasma potential profiles present in front of an aperture in an argon plasma. The transmission of the electrons through a finite thickness aperture is also experimentally investigated and comparison is made with a single particle orbit model. It is found that the measurement of the transmission can be used as a diagnostic of the perpendicular energy of charged particles in the presence of a magnetic field.

\section{POTENTIAL MEASUREMENT IN FRONT OF THE SAMPLING APERTURE}

Plasma potential profile measurements in the front of a sampling aperture are of interest both for charged particle

\footnotetext{
a) On leave from Southwestern Institute of Physics, Leshan, Sichuan, People's Republic of China.
} 


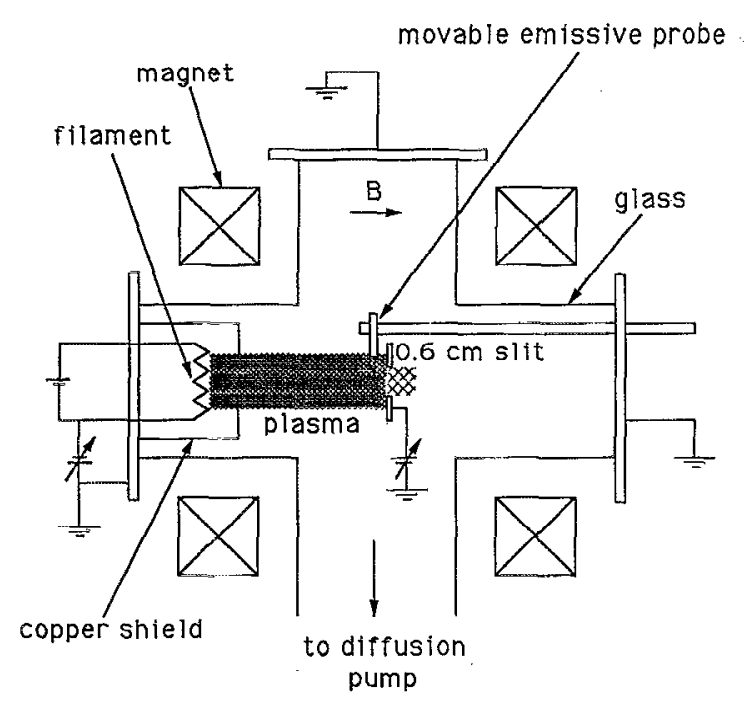

FIG. 1. Diagram of the plasma source and measurement system.

diagnostics in plasmas and for ion beam extraction in ion sources. The charged particle flux through the aperture depends on the potential profiles around the sampling aperture. The measured profile is the result of the interaction between the plasma and the solid surfaces.

Experiments were carried out in a filament discharge device shown in Fig. 1. Base pressure of the device was $10^{-6}$ Torr and the operating pressure was $(1-4) \times 10^{-4}$ Torr. Typical argon plasma densities varied from $10^{7}$ to $10^{8} \mathrm{~cm}^{-3}$. The electron temperature $T_{e}$ was $1-3 \mathrm{eV}$. The magnetic field was varied from 0 to $60 \mathrm{G}$. The emissive probe $^{2}$ used as a plasma potential indicator was of tungsten, $0.02 \mathrm{~cm}$ in diameter, $0.5 \mathrm{~cm}$ in length. It was connected to copper support wires which were insulated from the plasma by ceramic. A plate normal to the $B$ field had a $0.6-\mathrm{cm}$ slit and the emissive probe filament was parallel to the slit. The slit. was oriented perpendicular to the discharge filaments which were spaced less than the ionizing electron gyroradius. Plasma potential profiles in the front of the slit were measured by the floating point of the emissive probe operated in strong emission. Probe scans were made across the slit.

Figures $2(a), 2(b)$, and $2(c)$ show two-dimensional equipotential contours of the electric potential distribution in front of the slit in the plates with bias voltages $+5,0$, $-10 \mathrm{~V}$, respectively. The measurements were made in a 4$\mathrm{G}$ magnetic field. It is apparent that potential profile distortions appear when the slit plate was biased negatively or zero, while the potential distortions disappear when the slit plate is biased positively. The detailed characteristics of the plasma potential profile in front of the slit depends on the boundary condition of the plate. The aperture voltage affects the energy distribution of electrons passing through it. $E_{\|}$decelerates electrons, while in case of zero or negative bias, $E_{1}$ results in a transverse diffusion of electrons through electromagnetic drift effects.
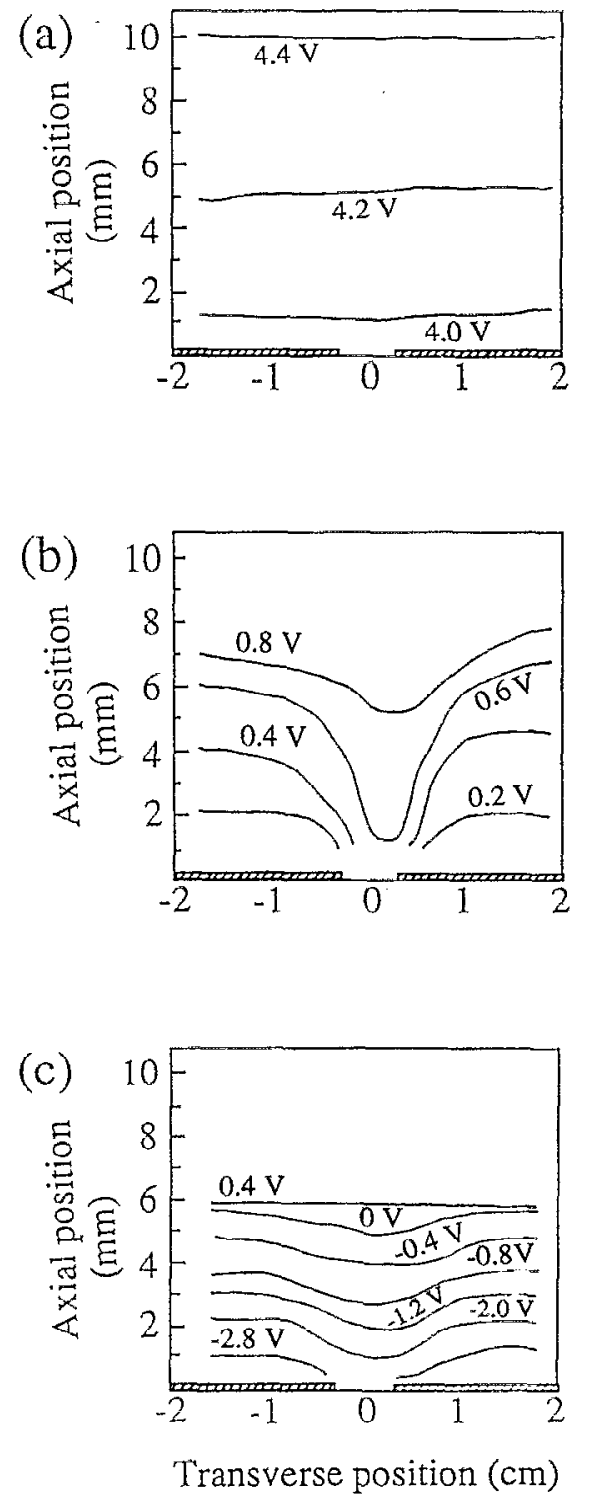

FIG. 2. Potential contours in front of the aperture. (a), (b), and (c) correspond to aperture plate biases of $+5,0$, and $-10 \mathrm{~V}$, respectively.

\section{TRANSMISSION MEASUREMENTS THROUGH AN APERTURE AND ITS ANALYSIS}

Here we investigate the transmission of an uniform electron beam through a finite thickness aperture. A schematic diagram of the experimental setup is shown in Fig. 3. The electron emitter is a $0.003-\mathrm{cm}$ wire thoriated tungsten mesh $3 \mathrm{~cm}$ by $6 \mathrm{~cm}$, and the spacing between filaments is $0.03 \mathrm{~cm}$. The mesh is negatively biased at $2.5-4.5 \mathrm{~V}$ with respect to the grounded grid. The emitted electrons are accelerated parallel to the magnetic field to an energy of $2.5-4.5 \mathrm{eV}$. A $0.3 \mathrm{~cm}$ in diameter 3-cm-long cylindrical aperture with a movable collector is also shown in Fig. 3. The collector consists of a plane electrode with its axis parallel to magnetic field line. The collector can be slid parallel to the $B$ field along the axis of the outer cylinder. Varying the collector position effectively changes the aperture thickness.

$\mathrm{Krawec}^{3}$ had given an analysis of the problem for both 


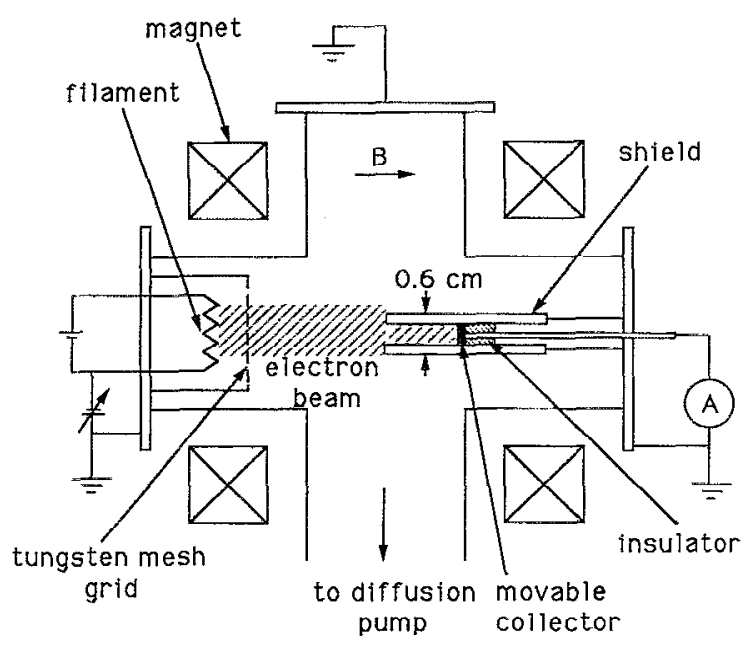

FIG. 3. Experimental setup of the transmission measurement.

thick and thin apertures. Staudenmaier et al. ${ }^{4}$ introduced a method to determine the ion temperature of deuterium and metal impurities based on the measurements of ion flux transmission of an aperture. In this paper, we consider the transmission process theoretically, based upon a single particle orbit model. Collision and space charge effects are ignored.

A calculation of the current collected through an aperture of radius $R$ can be used to determined the perpendicular energy of the charged particles. Consider a uniform distribution of charged particles with a given $r_{\perp}$ and with a velocity parallel to the magnetic field equal to $v_{z}$. The collected current consists of two parts. The first part $I_{1}$, which corresponds to those charged particles whose gyro-orbits will not hit the aperture wall, is collected as a constant current by the collector at any position along the aperture. The second part $I_{2}$ corresponds to those charged particles whose gyro-orbits may intercept the aperture wall but are collected before the interception occurs. The current collected by the collector when it is placed at the aperture entrance is $I_{0}$. The uniformity of the spatial distribution of the charged particles gives

$$
I_{0}=q n_{0} v_{z} \pi R^{2}
$$

where $q, n_{0}$, and $v_{z}$ (assumed to be a constant) are the charge, number density, and axial velocity of the charged particle respectively. After the collector is moved away from the entrance, it will collect both $I_{1}$ and $I_{2}$.

The charged particles have a perpendicular velocity distribution function which can be written

$$
f\left(v_{\perp}\right)=m / 2 \pi T_{\perp} \exp \left(-m v_{\perp}^{2} / 2 T_{\perp}\right),
$$

where $m, v_{\perp}$ and $T_{\perp}$ are the mass, perpendicular velocity, and perpendicular temperature (in $\mathrm{eV}$ ) of the charged particles.

The calculation of $I_{1}$ is straightforward. Referring to Fig. 4(a)

$$
I_{1}=\int_{0}^{R} q n_{0} v_{z} \pi\left(R-r_{\perp}\right)^{2} f\left(v_{\perp}\right) 2 \pi v_{\perp} d v_{\perp} .
$$

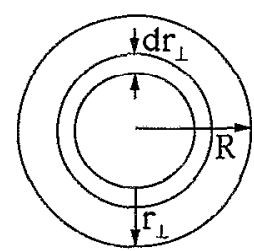

(a)

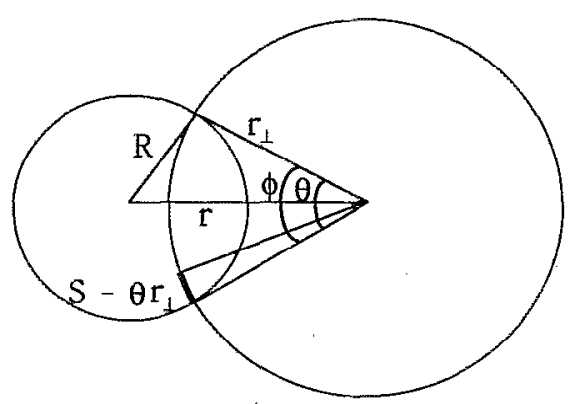

(b)

FIG. 4. (a) Geometry used to derive Eq. (5), (b) geometry used to derive Eq. (11).

After simplification, this expression becomes

$I_{1}=\int_{0}^{1} 2 \pi v_{z} R^{2} q n_{0} \sigma^{2}(1-X)^{2} \exp \left(-\sigma^{2} X^{2}\right) X d X$,

where $X \equiv r_{\perp} / R$ and $\sigma^{2} \equiv m \omega_{c}^{2} R^{2} / 2 T_{1}, \omega_{c}$ is the particle gyrofrequency. A transmission function for the aperture can be defined by dividing Eq. (2) by $I_{0}$. This results in

$$
\begin{aligned}
I_{1} / I_{0} & =\frac{I_{1}}{q n_{0} \pi v_{z} R^{2}} \\
& =2 \sigma^{2} \int_{0}^{1}(1-X)^{2} \exp \left(-\sigma^{2} X^{2}\right) X d X .
\end{aligned}
$$

The solid lines in Fig. 5 gives the results of numerical calculations of $I_{1} / I_{0}$ as a function of electron perpendicular temperature $T_{1}$ at different $B$ fields. The calculation of $I_{2}$ is more complicated. Referring to Fig. 4 (b), let $S$ be the arc length of the particle gyro-orbit projected on the aperture. The orbital angle $\theta$, through which each particle rotates while traversing an aperture length $z$, is given by

$$
\theta=\omega_{c} z / v_{z^{*}} \cdots
$$

This corresponds to motion through an arc length $\theta r_{1}$. Therefore, the probability of the particle of gyro-radius $r_{\perp}$ entering the aperture and being collected is $(\phi-\theta) / 2 \pi$. Let $r$ be the distance between the centers of the aperture and the gyro-center of the particle. Clearly

$$
\phi=2 \arccos \left[\left(r^{2}+r_{1}^{2}-R^{2}\right) / 2 r r_{1}\right],
$$

$\phi-\theta>0$ implies $r_{u}>r>r_{\perp}$, where

$$
\begin{aligned}
& r_{u}=r_{\perp} \cos (\theta / 2)+\sqrt{R^{2}-r_{\perp}^{2}\left[\sin ^{2}(\theta / 2)\right]}, \\
& r_{l}=r_{1} \cos (\theta / 2)-\sqrt{R^{2}-r_{\perp}^{2}\left[\sin ^{2}(\theta / 2)\right]} .
\end{aligned}
$$




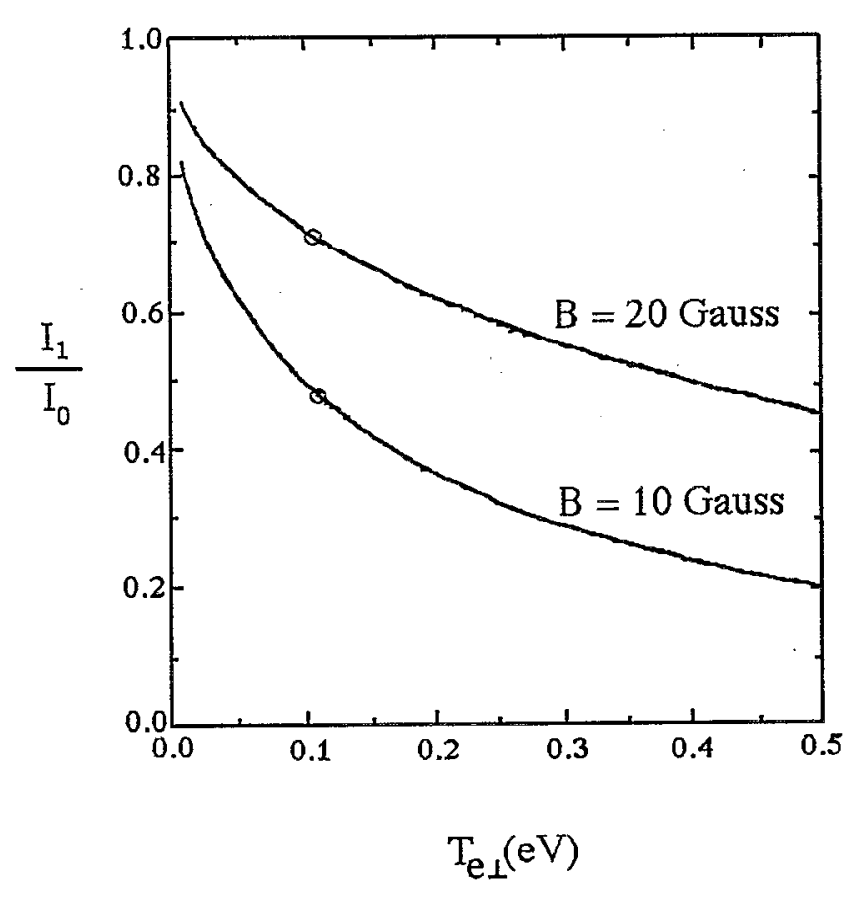

FIG. 5. $I_{1} / I_{0}$ as a function of perpendicular electron temperature at $B=20 \mathrm{G}$ and $B=10 \mathrm{G}$. Experimental data are indicated by " $O$ ".

Based on the geometry in Fig. 4(b), $I_{2}$ is given by

$$
I_{2}=\int_{0}^{\infty} \int_{r_{l}}^{r_{u}} f\left(v_{1}\right) q n_{0} v_{z}(\phi-\theta) \overrightarrow{d u_{1}} r d r
$$

Let

$$
G(\phi, \theta) \equiv \begin{cases}0, & \text { if } \phi-\theta \leqslant 0 \\ (\phi-\theta), & \text { if } \phi-\theta>0\end{cases}
$$

The characteristic of $G(\phi, \theta)$ allows us to change the integration limits $r_{u}$ and $r_{l}$ to $\left(R+r_{\perp}\right)$ and $\left|R-r_{\perp}\right|$, respectively. Dividing $I_{2}$ by $I_{0}$, we have

$$
\begin{aligned}
I_{2} / I_{0}= & \int_{0}^{\infty} \int_{|1-X|}^{1+X} \frac{m \omega_{c}^{2} R^{2}}{\pi T_{1}} \\
& \times \exp \left(-\sigma^{2} X^{2}\right) X Y G(\phi, \theta) d X d Y,
\end{aligned}
$$

where $Y=r / R$.

In Fig. $6, I / I_{0} \equiv I_{1} / I_{0}+I_{2} / I_{0}$ is calculated as a function of aperture thickness $z$. $I / I_{0}$ decreases to $I_{1} / I_{0}$ as indicated by the solid line.

In the previous derivation, we have assumed a constant parallel charged particle velocity $v_{z}$ with $E_{z}=1 / 2 m v_{z}^{2}$, corresponding to a delta distribution function $\delta\left(v-v_{z}\right)$. For arbitrary energy distribution function $f\left(E_{z}\right)$, the values of $I_{0}, I_{1}$, and $I_{2}$ are changed to $I_{0}^{\prime}, I_{1}^{\prime}$, and $I_{2}^{\prime}$ respectively. Correspondingly, Eqs. (5) and (11) become

$$
I_{1}^{\prime} / I_{0}^{\prime}=\frac{\int_{0}^{\infty} I_{1} f\left(E_{z}\right) v_{z} d v_{z}}{\int_{0}^{\infty} I_{\alpha} f\left(E_{z}\right) v_{z} d v_{z}},
$$

and

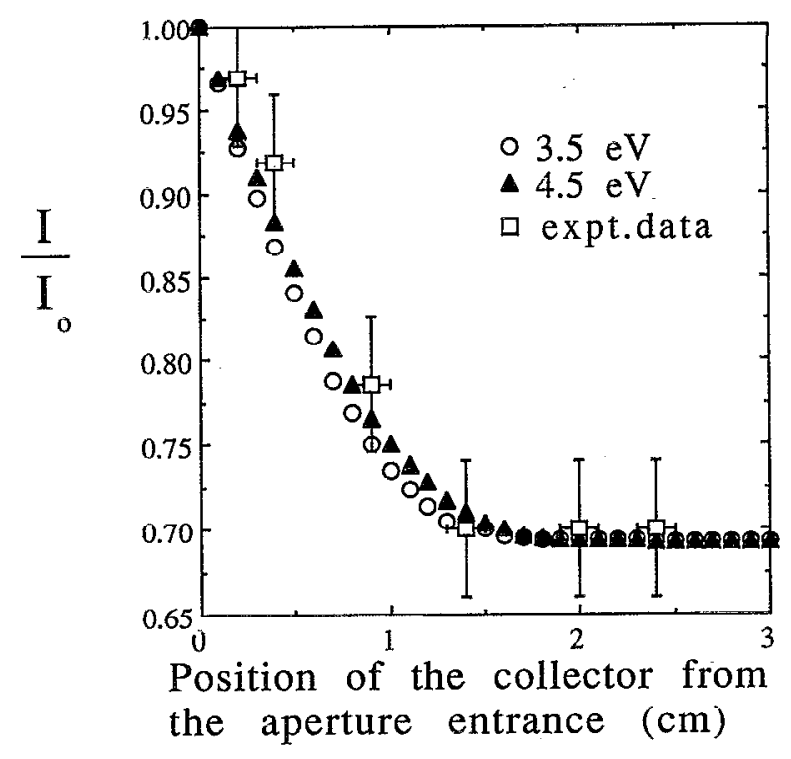

FIG. 6. $I / I_{0}$ as a function of position, (i.e., thickness of aperture) at $B=20 \mathrm{G}$, Beam average energy $=3.5 \mathrm{eV}$. The square points are experiment data. The solid lines are theoretical results.

$$
I_{2}^{\prime} / I_{0}^{\prime}=\frac{\int_{0}^{\infty} I_{2} f\left(E_{z}\right) v_{z} d v_{z}}{\int_{0}^{\infty} I_{0} f\left(E_{z}\right) v_{z} d v_{z}} .
$$

It is found that the results for $I_{1}^{\prime} / I_{0}^{\prime}, I_{2}^{\prime} / I_{0}^{\prime}$ and $I_{1} / I_{0}$, $I_{2} / I_{0}$ are essentially unchanged (see Fig. 7 ) if the parallel charged particle energies are assumed to have a shifted Gaussian distribution with mean energy equal to $E_{z}$ and a standard deviation $\delta$ as large as $0.5 E_{z}$. The data show that for $\delta=0.75 E_{z}$, there is only a slight shift. For larger $\delta$, larger deviations are observed but $I / I_{0}$ values are still within $5 \%$ of the value for $\delta=0$.

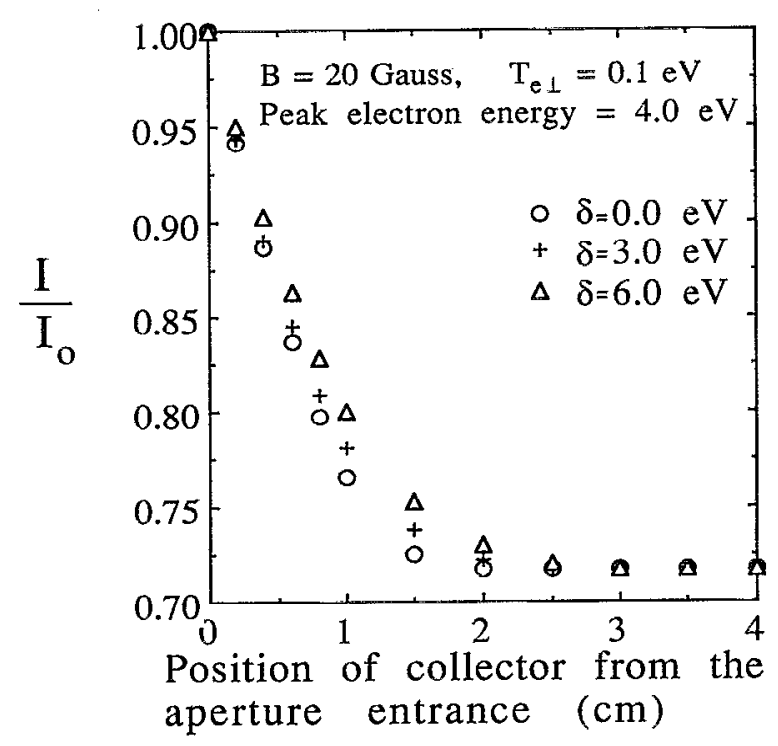

FIG. 7. $I / I_{0}$ as a function of position of the collector for different values of standard deviation $\delta$ at a fixed peak parallel electron energy $E_{z}=4.0$ $\mathrm{eV}$. 
We have made a comparison between experimental values of $I / I_{0}$ for electrons and the single particle orbit transmission model. The experimental data in Fig. 6 give the measurements of the transmission parameter, $I / I_{0}$, as a function of the position of the collector in a $20 \mathrm{G} B$ field. Here, $I_{0}$ is the total entrance current collected at the $z=0$ position. At this position, the collector front surface coincides with the front plane of the cylindrical aperture. The results of the theoretical calculation for similar parameters are also shown as the solid curve in Fig. 6. Agreement is good except at small $z$ at which $I / I_{0}$ is uncertain because of experimental inaccuracies in the collector positions.

Figure 6 shows the transmission $I / I_{0}$ as a function of electron temperature at a different magnetic $B$ field. The solid lines are computed from Eq. (5) and the points are experimental results. From the data shown in Fig. 6 the electron temperature is estimated to be approximately 0.12 $\mathrm{eV}$. This value agrees with the mesh temperature measured by an optical pyrometer of $0.1 \pm 0.02 \mathrm{eV}$.

\section{DISCUSSION AND CONCLUSIONS}

A single particle orbit model is shown to be consistent with the transmission experiments with electrons. Particle gyro-motion in the magnetic fields governs the character of aperture transmission. Therefore, the selection of the aperture size, $B$ field, and temperature is of importance for optimum experimental conditions. The transmission experiments were performed with an electron beam of low num- ber density. Agreement of the theoretical and experimental results suggests that a cylindrical aperture with a movable grounded collector can be used as a diagnostic device to measure the perpendicular energy of electrons. The extension of this diagnostic device to measure perpendicular ion energy in plasma is also possible. In principle, by adding grids in front of the cylindrical aperture, we can modify this diagnostic device to measure both the parallel and perpendicular ion energy. By proper bias, the electron current can be made negligibly small compared to the ion current. This suggests the possibility of obtaining information about the ion energy directly from the collected current characteristic. When we consider the aperture effect in a plasma, rather than in vacuum, potential profile distortion must also be taken into account. Proper bias of the aperture can reduce this problem.

\section{ACKNOWLEDGMENTS}

This work was supported by U.S. DOE grant DEFG02-88ER553264 U. S. NSF grant ECS-8704529 and a gift from Varian Assoc.

${ }^{1}$ H. W. Drawin, in Plasma Diagnostics, edited by W. Lochte-Holgreven (North-Holland, Amsterdam, 1968), pp. 777-841.

${ }^{2}$ Noah Hershkowitz, Plasma Diagnostics, Vol. 1, edited by O. Auciello and D. L. Flamm (Academic, New York, 1989).

${ }^{3}$ R. Krawec, NASA TN D-5746, 1976.

${ }^{4} \mathrm{G}$. Staudenmaier, P. Staib, and W. Poschenrieder, J. Nucl. Mater. 93 \& 94, 121 (1980). 